\title{
Improved microbubble (MB) Localisation Using Particle Detecting algorithm: Evaluation of Algorithm Performance for Different Beamforming Methods
}

Voulgaridou, Vasiliki ; Nicola, Barbara; McDougal, Steven; Arthur , Lachlan; Kanoulas , Evangelos; Lu , Weiping; Diamantis , Konstantinos; Jensen, Jørgen Arendt; Sboros, Vassilis

Published in:

2020 IEEE International Ultrasonics Symposium

Link to article, DOI:

10.1109/IUS46767.2020.9251433

Publication date:

2020

Document Version

Peer reviewed version

Link back to DTU Orbit

Citation (APA):

Voulgaridou, V., Nicola, B., McDougal, S., Arthur , L., Kanoulas, E., Lu , W., Diamantis, K., Jensen, J. A., \& Sboros, V. (2020). Improved microbubble (MB) Localisation Using Particle Detecting algorithm: Evaluation of Algorithm Performance for Different Beamforming Methods. In 2020 IEEE International Ultrasonics Symposium IEEE. https://doi.org/10.1109/IUS46767.2020.9251433

\section{General rights}

Copyright and moral rights for the publications made accessible in the public portal are retained by the authors and/or other copyright owners and it is a condition of accessing publications that users recognise and abide by the legal requirements associated with these rights.

- Users may download and print one copy of any publication from the public portal for the purpose of private study or research.

- You may not further distribute the material or use it for any profit-making activity or commercial gain

- You may freely distribute the URL identifying the publication in the public portal 


\section{Improved microbubble (MB) Localisation Using Particle Detecting algorithm: Evaluation of Algorithm Performance for Different Beamforming Methods}

\author{
Vasiliki Voulgaridou \\ School of Engineering and \\ Physical Sciences \\ Heriot Watt Univeristy \\ Edinburgh, UK \\ vv17@hw.ac.uk \\ Barbara Nicolas \\ Univ Lyon, INSA - \\ Lyon, Université Claude \\ Bernard Lyon 1, UJM-Saint \\ Etienne, CNRS, Inserm, \\ CREATIS UMR 5220, \\ U1206, F-69621, \\ LYON, France \\ barbara.nicolas@creatis.insa \\ -lyon.fr \\ Weiping $\mathrm{Lu}$ \\ School of Engineering and \\ Physical Sciences \\ Heriot Watt Univeristy \\ Edinburgh, UK \\ w.lu@hw.ac.uk \\ Konstantinos Diamantis \\ Institute for Digital \\ Communications \\ University of Edinburgh \\ Edinburgh, UK \\ kdiamantis84@gmail.com
}

\author{
Steven McDougall \\ Institute of GeoEnergy \\ Enginering \\ Heriot Watt Univeristy \\ Edinburgh, UK \\ s.r.modougall@hw.ac.uk
}

\author{
Lachlan Arthur \\ School of Engineering and \\ Physical Sciences \\ Heriot Watt Univeristy \\ Edinburgh, UK \\ 1a25@hw.ac.uk
}

\author{
Evangelos Kanoulas \\ School of Engineering and \\ Physical Sciences \\ Heriot Watt Univeristy \\ Edinburgh, UK \\ ek28@hw.ac.uk
}

\author{
Jørgen Arendt Jensen \\ Center for Fast Ultrasound \\ Imaging, Dept. of Health \\ Technology, \\ Technical University of Denmark \\ Lyngby, Denmark \\ jaje@dtu.dk
}

\author{
Vassilis Sboros \\ School of Engineering and \\ Physical Sciences \\ Heriot Watt Univeristy \\ Edinburgh, UK \\ vs148@hw.ac.uk
}

\begin{abstract}
The performance of image analysis techniques (particle detection) on contrast enhanced ultrasound (CEUS) images could be enhanced in combination with the use of the right beamformer (BF). The current study investigates the best performing combination of a particle detecting algorithm (Kanoulas et al. 2019) with four beamformers (BFs), classical and adaptive. In a series of in silico experiments, adjacent MBs are placed in distances comparable to the lateral resolution limit, the CEUS images of the MBs were simulated in FieldII, and finally beamformed with the four methods. The images were processed with the MB detection algorithm and the results were evaluated by the true detections (TD), missed MBs, spurious detections, and localisation uncertainty (LU). For the smallest distances all methods deteriorate but the MV methods provided 4-12\% more TD. For the intermediate distances the TD were comparable for all BFs but the adaptive methods provided lower $\mathrm{LU}$. When a set of evaluation metrics is used, the adaptive methods provide marginally but systematically improved results which suggests that, under the appropriate imaging conditions, they could be used to enhance vessel mapping.
\end{abstract}

Keywords-medical, beamforming, resolution, imageprocessing, microbubble tracking

\section{INTRODUCTION}

CEUS images can be processed with the particle detecting algorithm based on Kanoulas et al. 2019 [1] that provides an estimation of microbubble (MB) position and allows the identification of sub-resolution vessel structures in the image. The input image data quality affects both the accuracy of detection and localisation which in turn will affect the vessel mapping. Classically the CEUS image data are generated with conventional beamformers and it is unclear whether adaptive beamformers, that provide improved lateral resolution and potentially MB localisation [2]-[5], will also provide improved super-resolution vessel mapping results. It is this association between beamforming and vessel accuracy in super-resolution ultrasound imaging that has not been previously investigated. The aim of this work is to investigate MB detection and localisation performance when they are located within adjacent vessels in distances around the resolution limit.

Specifically, the four BF methods, namely the DAS with a uniform apodisation window (called DAS boxcar in the following), DAS with a Hanning apodisation window, MV in the frequency domain, and MV in the time domain [6] were chosen in this investigation. In a series of in silico experiments the MBs were placed in vessels at various positions close to the lateral resolution limit. The US images of the MBs were 
simulated in Field II [7, 8], the four different BFs were implemented, and the resulting images were later processed with the MB detection algorithm. The accuracy of detection and localisation can be evaluated when the ground truth (GT) position of the MBs is compared with the estimated.

\section{METHODS}

\section{A. Experimental design}

Lateral resolution is typically evaluated with two stationary point spread functions (PSFs) at the same depth, in our case MBs. However, resolving adjacent vessels is a slightly different problem. The relative positions of the MBs in motion may impact vessel reconstruction in a complex manner. It is this problem that is investigated here. The phantom used for the in silico experiments, Fig.1, consists of two linear vessels(hollow cylindrical elements) with a diameter of $50 \mu \mathrm{m}$, in which MBs flow at opposite directions with the same constant flow rate of $5^{-10} \mathrm{~m}^{3} / \mathrm{s}$ following Poiseuille's law [9]. The opposite flow is achieved placing the inlet of the left vessel at the bottom and the inlet of the right vessel at the top. The MBs are injected randomly at each of the inlets of the two-vessel system with an injection interval for the system of $1 \mathrm{MB}$ every $0.1 \mathrm{sec}$. This allows the lateral signal interaction of $\mathrm{MBs}$ for the following cases: one in each vessel, one $\mathrm{MB}$ in one vessel and a cluster of MBs in the other, or clusters of MBs at each vessel.

\section{B. US imaging and beamforming}

The MB flow simulation provides the temporal and spatial coordinates of each MB. In addition to that, every MB has a unique ID and can be followed throughout its path in a vessel. The spatial information of the MBs for 100-time frames was used as input in a FieldII imaging simulation $[7,8]$ to generate a video sequence of flowing MBs. For the imaging simulation the excitation pulse was generated by the method of synthetic aperture of one active element, with pulse repetition frequency at $100 \mathrm{~Hz}$ and image depth between $52-80 \mathrm{~mm}$. The US images were created with four BF methods: DAS boxcar, DAS

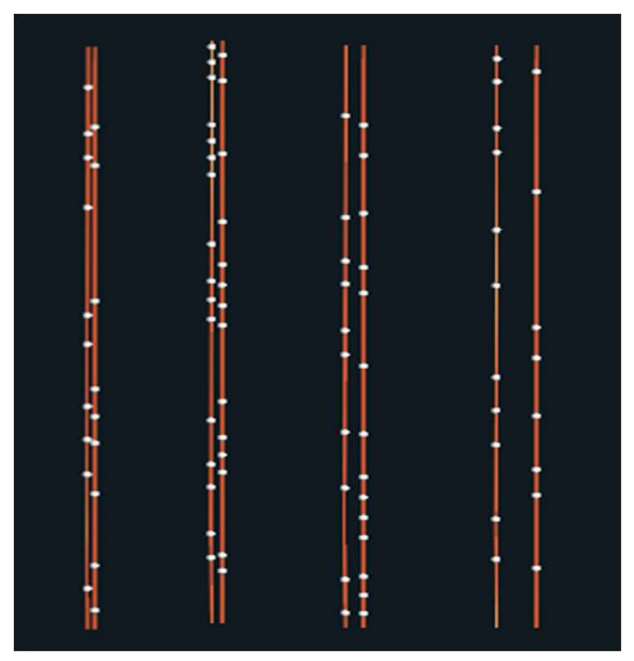

Fig. 2: Four different vessel separations. MBs (white dots) are flowing through the vessels.
Hanning, MV in the frequency domain, and MV in the temporal domain, Fig.2, [10].

\section{Detection, Localisation and Evaluation of results}

The simulated US images were processed with the MB identification algorithm. The MB detection algorithm first determines the particle regions classifying them as MB (area) or background (noise) based on an intensity thresholding. The MB regions are segmented, with marker-controlled watershed segmentation [11], providing the MB segments. Using a series of criteria such as the peak intensity, the minimum and the maximum size of the segments, the segments of MBs are selected. Then, using the intensity weighted centre of mass (COM) method, the algorithm provides the estimated location of each MB.

The assessment of the detection and localisation can be done quantitatively using the following statistics:

- True detection (TD) rate: percentage of correct detections that correspond to a GT MB

- Spurious events rate: percentage of false detections that do not correspond to GT MBs

- Missed events rate: percentage of GT MBs that were not matched with a detection and

- Localisation Uncertainty (LU): It is the calculated root mean square error of the distance of each localisation from its true MB location. This applies only to the paired MBs (algorithm true detection and GT MBs) and is a measure of the localisation uncertainty (LU).

\section{RESULTS}

Table I shows the percentage of total MBs that was correctly detected by the algorithm for the 4 BFs. The increase of the lateral vessel separation correlates with an increase in the number of correct detections. At the largest separation $(1000 \mu \mathrm{m})$ all the MBs are detected. This indicates that for those separations and above all beamforming methods provide the same detection performance, which is due to the fact that at $1000 \mu \mathrm{m}$ the MB echoes are well separated and laterally independent. For the
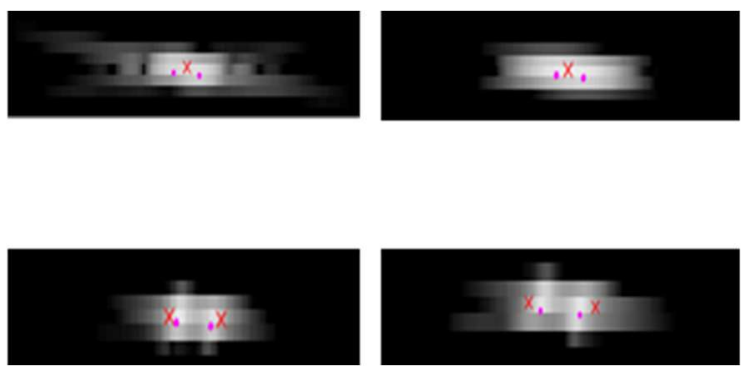

Fig. 1 The GT position of the MBs (purple dot) and the position of the MB as was detected from the algorithm (red ' $x$ ') for a vessel separation of

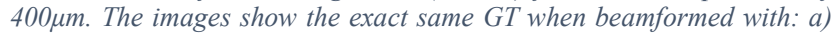
DAS boxcar, b) DAS Hanning, c) MV Subband, d) MV temporal. 
smallest separation where there is a significant PSF overlap, a large percentage of the MBs remains undetected. This percentage reaches more than $20 \%$ for the $100 \mu \mathrm{m}$ separation. For these vessel separations, the MV methods are performing generally slightly better than the DAS, particularly the MV Temporal which outperform the DAS with a TD rate of $88 \%$ for the $100 \mu \mathrm{m}$ separation. In addition, the quality of detections is also determined by the percentage of spurious events and by the LU. The spurious events, Table II, for all BF methods and vessel separations were generally low. The methods have comparable and low values of LUs for separations above $1000 \mu \mathrm{m}$ which is due to minimal PSF interference at those distances. It is important to note that the LU provides a useful information only for cases where the TD rate is already satisfactory. Indeed, for the smaller separations, when the TD rate is low, the MBs are so close to one another that the misplaced detections are often in the centre of the vessels leading to a relatively small LU that is not representative of the algorithm performances.

\section{DISCUSSION}

The results of this experiment do not show a significant difference in MB detections results when the MV methods are compared to the DAS methods but they show a systematically better performance of the MV methods either in the form of TD rate or LU when compared to the DAS. This systematic performance, even though modestly better, suggests that the combination of MV methods with the detection algorithm may provide more accurate vessel mapping under the appropriate imaging conditions.

A representative performance assessment of the different beamformers can only be done using a set of evaluation metrics, with the true detections being the primary one. The spurious events in these noise-free experiments are generally low therefore the use of this metric would not provide additional value but in a more realistic setting, with noise, this metric could provide valuable information The experiment suggests that the most informative combination of metrics would be the true detections along with the localisation uncertainty. Fig.2 shows that for overlapping PSFs the detections could be either between the MBs or outside the 2-vessel region, which is the reason for deterioration of the LU in intermediate vessel distances.

table I. True Detection Rate By Vessel Separation

\begin{tabular}{|l|c|c|c|c|}
\hline \multirow{2}{*}{} & \multicolumn{4}{|c|}{ Percentage of MBs that were correctly detected (\%) } \\
\cline { 2 - 5 } & $\mathbf{1 0 0} \boldsymbol{\mu m}$ & $\mathbf{2 0 0} \boldsymbol{\mu m}$ & $\mathbf{4 0 0 \mu \boldsymbol { m }}$ & $\mathbf{1 0 0 0} \boldsymbol{\mu m}$ \\
\hline DAS Boxcar & 76 & 88 & 91 & 100 \\
\hline DAS Hanning & 76 & 85 & 90 & 100 \\
\hline MV Subband & 80 & 87 & 93 & 100 \\
\hline MV Temporal & 88 & 92 & 93 & 100 \\
\hline
\end{tabular}

TABLE II. SPuRious Detection RATE By VesSEL SEPARATion

\begin{tabular}{|l|c|c|c|c|}
\hline \multirow{2}{*}{} & \multicolumn{4}{|c|}{ Percentage of spurious detections (\%) } \\
\cline { 2 - 5 } & $\mathbf{1 0 0 \mu \boldsymbol { m }}$ & $\mathbf{2 0 0} \boldsymbol{\mu m}$ & $\mathbf{4 0 0} \boldsymbol{\mu m}$ & $\mathbf{1 0 0 0} \boldsymbol{\mu m}$ \\
\hline DAS Boxcar & 1.17 & 1.32 & 0.26 & 1.05 \\
\hline DAS Hanning & 0.02 & 0.1 & 0.02 & 0.04 \\
\hline MV Subband & 0.06 & 0.08 & 0.45 & 0 \\
\hline MV Temporal & 0 & 0.02 & 0.16 & 0.43 \\
\hline
\end{tabular}

TABLE III. LOCALISATION UnCERTAINTY By Vessel SEPARATION

\begin{tabular}{|l|c|c|c|c|}
\hline \multirow{2}{*}{} & \multicolumn{4}{|c|}{ Localisation Uncertainty $(\boldsymbol{\mu m})$} \\
\cline { 2 - 5 } & $\mathbf{1 0 0 \mu \boldsymbol { m }}$ & $\mathbf{2 0 0} \boldsymbol{\mu m}$ & $\mathbf{4 0 0 \mu m}$ & $\mathbf{1 0 0 0} \boldsymbol{\mu m}$ \\
\hline DAS Boxcar & 155 & 169 & 180 & 146 \\
\hline DAS Hanning & 145 & 164 & 178 & 139 \\
\hline MV Subband & 135 & 140 & 121 & 120 \\
\hline MV Temporal & 149 & 144 & 141 & 149 \\
\hline
\end{tabular}

\section{CONCLUSIONS}

The MV BFs improves the percentage of correct MB detections for small lateral separations while for larger separations all the $\mathrm{BF}$ methods provide comparable true detections. In the intermediate vessel separations, the benefit of using adaptive $\mathrm{BF}$ is shown in the accuracy of the MB localisation.

The future work could include a study of the performance for various imaging settings under different noise levels. Furthermore, the data of this experiment could be used and assessed in the context of vessel mapping.

\section{ACKNOWLEDGMENT}

The authors would like to acknowledge the work of Dr Ahmed Boujelben whose software has been used for the phantom design. Also, Steven McDougall would like to acknowledge support from EPSRC SofTMech Grant $\mathrm{EP} / \mathrm{N} 014642 / 1$.

\section{REFERENCES}

[1] Kanoulas E, Butler M, Rowley C, Voulgaridou V, Diamantis K, Duncan WC, et al. Super-Resolution Contrast-Enhanced Ultrasound Methodology for the Identification of In Vivo Vascular Dynamics in 2D. Investigative radiology. 2019;54(8):500-16.

[2] J.F.Synneva ${ }^{\circ}$,A.Austeng,andS.Holm, “Adaptivebeamforming applied to medical ultrasound imaging,” IEEE Trans. Ultrason., Fer-roelectr., Freq. Control, vol. 54, no. 8, pp. 1606-1613, Aug.2007.

[3] I. K. Holfort, F. Gran, and J. A. Jensen, "Broadband minimum variance beamforming for ultrasound imaging," IEEE Trans. Ultrason., Ferro-electr.,Freq.Control,vol.56,no.2,pp.314-325,Feb.2009.

[4] K. Diamantis, I. H. Voxen, A. H. Greenaway, T. Anderson, J. A. Jensen, and V. Sboros, "A comparison between temporal and subband 
minimum variance adaptive beamforming," in Proc. SPIE Med. Imag., vol. 90400L, Mar. 2014. [Online]. Available:10.1117/12.2043602.

[5] K. Diamantis, A. Greenaway, T. Anderson, J. A. Jensen, and V. Sboros, "Experimental performance assessment of the sub-band minimum vari-ance beamformer for ultrasound imaging," Ultrasonics, vol. 79, pp. 87-95,2017.

[6] K. Diamantis, M. Butler, T. Anderson, J. A. Jensen and V. Sboros, "Minimum Variance beamforming for closely spaced microbubbles," 2019 IEEE International Ultrasonics Symposium (IUS), Glasgow, United Kingdom, 2019, pp. 571-574, doi: 10.1109/ULTSYM.2019.8925587.

[7] Jensen JA, Svendsen NB. Calculation of pressure fields from arbitrarily shaped, apodized, and excited ultrasound transducers. IEEE Transactions on Ultrasonics, Ferroelectrics, and Frequency Control. 1992;39(2):262-7.

[8] Jensen JA. Field: A Program for Simulating Ultrasound Systems. Medical and Biological Engineering and Computing. 1997;34(sup. 1):351-
3.Jensen, J.A., Field: A Program for Simulating Ultrasound Systems. Medical and Biological Engineering and Computing, 1997. 34(sup. 1): p. 351-353

[9] Boujelben A, Watson M, McDougall S, Yen Y-F, Gerstner ER, Catana C, et al. Multimodality imaging and mathematical modelling of drug delivery to glioblastomas. 2016.

[10] Diamantis K, Anderson T, Butler MB, Villagomez-Hoyos CA, Jensen JA, Sboros V. Resolving Ultrasound Contrast Microbubbles Using Minimum Variance Beamforming. IEEE Trans Med Imaging. 2019;38(1):194-204

[11] Yang, X., H. Li, and X. Zhou, Nuclei Segmentation Using MarkerControlled Watershed, Tracking Using Mean-Shift, and Kalman Filter in Time-Lapse Microscopy. IEEE Transactions on Circuits and Systems I: Regular Papers, 2006. 53(11): p. 2405-2414. 